\title{
DUALITY IN VECTOR LATTICES
}

\author{
D. B. LOWDENSLAGER ${ }^{1}$
}

Introduction. We shall prove an abstract theorem on the duality of vector lattices. It is powerful and general enough to yield quickly the duality of both the classical examples of concrete vector lattices as well as various abstract analogs of these lattices. The theorem treats the duality of $L_{1}$ and $L_{\infty}$ so as to exhibit the relation of their duality to the duality of the $L_{p}$ spaces for $1<p<\infty$. The proof itself is elementary in that it uses no representation theory for lattices and no facts about weak compactness. Let $L$ be a complete vector lattice; $f$ will be called $o$-continuous $[4$, p. 23] if $f$ is a real valued linear functional on $L$ such that whenever $\left\{x_{\alpha} \mid \alpha \in A\right\}$ is a net in $L$ with $\Lambda_{\beta} \bigvee_{\alpha>\beta} x_{\alpha}=\bigvee_{\beta} \Lambda_{\alpha>\beta} x_{\alpha}=x$, then $\lim f\left(x_{\alpha}\right)=f(x)$. This implies that $f$ is bounded in the sense that the set $\{f(x): 0 \leqq x \leqq y\}$ is bounded for each $y$. It follows that $f$ and the zero functional have a supremum and infimum relative to the lattice of all bounded linear functionals $[1$, p. 245].

The concepts of completeness and $o$-continuity arise naturally in a duality theory for this reason: the set $B$ of all bounded linear functionals on a vector lattice is easily seen to be a complete vector lattice, a rising (directed upward) set of linear functionals converging pointwise to its supremum; the same is true for the subset of $B$ consisting of the functions continuous in a topology $T$ such that if $U$ is a neighborhood of 0 , there is a neighborhood $V$ of 0 with $\{|x|: x \in V\}$ $C U$. In addition, if $x$ is an element in the original lattice, the functional on $B$ induced by $x$ is $o$-continuous.

We now give an important definition, which is followed by the main theorem. Recall that a nondegenerate bilinear functional on the cartesian product of two vector spaces $L$ and $M$, is one such that the maps $x \rightarrow x^{*}$ and $y \rightarrow y^{*}$ defined below are 1-1 onto their range.

Definition. $A$ pair of complete vector lattices, $(L, M)$ is said to be paired with respect to the nondegenerate bilinear functional $\langle x, y\rangle$ on $L \times M$ provided the functionals $y^{*}: x \rightarrow\langle x, y\rangle$ and $x^{*}: y \rightarrow\langle x, y\rangle$ are $o$ continuous for $x \in L, y \in M$, and each vector lattice is a sublattice of the lattice of all bounded linear functionals on the other, i.e., the functional

Received by the editors March 12, 1956 and, in revised form, August 27, 1956.

1 This paper is taken from the author's dissertation, University of Virginia, June 1955 , work on which was supported by a grant from the National Science Foundation.

The author owes his interest in this subject to E. J. McShane. 
$(y \vee 0)^{*}$ is the supremum of $y^{*}$ and 0 in the lattice of all bounded linear functionals on $L$, and a similar statement holds for $M$.

TheOREM. Let $(L, M)$ be a pair of complete vector lattices paired with respect to the nondegenerate bilinear functional $\{\langle x, y\rangle \mid x \in L, y \in M\}$. Every positive o-continuous linear functional on $M$ is the supremum of a rising collection of functionals of the form $x^{*}, x \in L$.

A proof of this theorem will follow after several preliminary remarks.

Definition. An ideal I of a complete vector lattice $L$ is a linear subspace of $L$ such that $x \in I,|y| \leqq|x|$ imply $y \in I$, and $D \subset I$ implies $\sup D \in I$, if $\sup D$ exists.

Lемма 1. The set of ideals of a complete vector lattice $L$ is a complete Boolean algebra under the natural inclusion ordering. Also $L$ is the direct sum of any ideal I and its Boolean complement $c I$; $c I$ is the set of $z$ such that $|z| \wedge|x|=0$ for all $x \in I$.

The proof of this lemma involves a routine use of vector lattice identities, for instance those in [1]. The lemma seems to appear first in [2]. What we have called ideals here were called "normal subspaces" by Bochner and Phillips, "closed $l$-ideals" by Birkhoff and "components" by Vulih. We shall use the words "complement," "union" and "intersection" to mean the Boolean operations on the ideals, and shall designate them by the symbols $c, \cup$, and $\cap$. Disjoint ideals will be ideals whose intersection is the zero ideal.

Lemma 2. Let $f$ be an o-continuous linear functional on a complete vector lattice $L$. Then $L$ is uniquely the direct sum of three disjoint ideals $P, N$, and $Z$, with the property that $f$ is strictly positive on $P$, strictly negative on $N$ and zero on $Z$. Let $x$ be the $P$ component of $z$ in $L$; then $(f \vee 0)(z)=f(x)$.

By a strictly positive linear functional $f$, we understand one such that $f(x)>0$ whenever $x>0$. This lemma is a version of the classical Hahn-Jordan result, and its proof is almost the same as that of a similar result in $[1$, p. 250]. $P$ is constructed as the set of $y$ such that $0<x \leqq|y|$ implies $f(x)>0, N$ as the set of $y$ with $0<x \leqq|y|$ implying $f(x)<0$ and $Z$ is the set of $y$ with $0<x \leqq|y|$ implying $f(x)=0$. We will call $P, N$, and $Z$ the positive, negative, and zero ideals, respectively, of $f$. It may be seen easily that the set of $o$-continuous functionals on $L$ is a sublattice of the lattice of all bounded linear functionals, and that two positive $o$-continuous functionals satisfy $f \wedge g=0$ if and only if their positive ideals are disjoint. This fact is used in the 
following lemma to prove that the Boolean algebras of ideals in $L$ and $M$ are intimately related. We mean by $|f|(x)$ the value of the functional $|f|$ at $x$.

Definition. Let $L$ be a complete vector lattice, $M$ a sublattice of the lattice of all o-continuous linear functionals on $L$, such that $D \subset M$, $D$ bounded above by $f \in M$ implies sup $D \in M$. Then

for any ideal $I$ of $L$, the perpendicular ideal to $I, p I$, is the ideal

$$
\{f: f \in M,|f|(x)=0 \text { for all } x \in I\} .
$$

for any ideal $J$ of $M$, the annihilated ideal, aJ, is the ideal

$$
\{x: x \in L, f(|x|)=0 \text { for all } f \in J\} .
$$

Lemma 3. Let $L$ and $M$ be as in the preceding definition. Then $p(a J)=J$, and $a$ satisfies $a\left(J_{1} \cup J_{2}\right)=a J_{1} \cap a J_{2}, a\left(J_{1} \cap J_{2}\right)=a J_{1} \cup a J_{2}$, and $a(c J)=c(a J)$.

Proof. Obviously $p I$ is an ideal; $a J$ is the intersection of the zero ideals of the elements of $J$, and is therefore an ideal. Moreover $p(a J) \supset J$, and if $g>0, g \in c J$, there exists $x>0$ in the positive ideal of $g ; x$ is in the zero ideal of every $f \in J$ by the remark after Lemma 2, and the construction of $c J$. Thus, by definition, $x \in a J$, and therefore $g \notin p(a J)$, which proves that $p(a J)=J$. A similar calculation shows that $a\left(J_{1} \cup J_{2}\right)=a J_{1} \cap a J_{2}$. Obviously $a\left(J_{1} \cap J_{2}\right) \supset a J_{1} \cup a J_{2}$; suppose $x>0, x \in c\left(a J_{1} \cup a J_{2}\right)$ but $x \in a\left(J_{1} \cap J_{2}\right)$. There exists $f>0, f \in J_{1}$, such that $f(x)>0$. Let $x=y+z$, where $y$ is in the positive ideal of $f, z$ in its complement. Since $y \in c\left(a J_{1} \cup a J_{2}\right)$, there is a positive $g \in J_{2}$ with $g(y)>0$. Then $y=u+v$ where $u$ is in the positive ideal of $g, v$ in its complement. Then $u$ is a strictly positive element in the positive ideals of both $f$ and $g$ so that $(f \wedge g)(u)>0$, and $u \in a\left(J_{1} \cap J_{2}\right)$, a contradiction. The third identity follows from the first two.

Proof of THEOREM. First we show that if $t$ is a positive $o$-continuous linear functional on $M$ satisfying $0<t \leqq x^{*}$ for some $x \in L$, there exists $u \in L$ such that $0<u^{*} \leqq t$. For there exists a scalar $r>0$ such that $r x^{*}-t$ is not positive, since otherwise inf $\left(r x^{*}-t\right)=-t \geqq 0$. Let $J$ be the negative ideal of $r x^{*}-t$ for such an $r$, and let $r x=u+v$, $u \in c(a J), v \in a J$. Then, for $0<f, f \in J$ we have $\langle r x, f\rangle=r x^{*}(f) \geqq 0$, while $\left(r x^{*}-t\right)(f)<0$, so that $0 \leqq f^{*}(r x)=f^{*}(u+v)=f^{*}(u) \leqq t(f)$. But for $f \in c J, f^{*}(u)=0$, by Lemma 3 . Thus $0 \leqq f(u) \leqq t(f)$ for all positive $f \in M$, and $0<u^{*} \leqq t$.

Now let $y$ be a positive $o$-continuous linear functional on $M$. The set of elements $v \in L$ satisfying $0 \leqq v^{*} \leqq y$ is directed upward, and these $v^{*}$ have a supremum $y_{0}$ in the set of all bounded linear func- 
tionals on $M$. If $y_{0} \neq y$, there is no element $u \in L$ such that $0<u^{*}$ $\leqq y-y_{0}$. If $\left(y-y_{0}\right) \wedge x^{*}=0$ for all positive $x \in L$, the positive ideal of $y-y_{0}$ would be in the zero ideal of each positive $x \in L$, which implies degeneracy of the bilinear form on $L \times M$. Let $x>0$ be such that $t=\left(y-y_{0}\right) \wedge x^{*}>0$; there is a $u \in L$ with $0<u^{*} \leqq y-y_{0}$ by the first part of the proof. This contradiction implies $y_{0}=y$, which completes the proof.

For our applications we need some additional information, contained in the following.

Proposition 1. Let $M$ be a sublattice of the set of all o-continuous linear functionals on the complete vector lattice $L$ such that $M$ distinguishes points of $L$ and $U \subset M, U \leqq f \in M$ implies sup $U \in M$. The following three conditions are equivalent.

1. $M$ is order convex (i.e., if $f \in M, g \in M$ and $f \leqq u \leqq g$ for some linear functional $u$, then $u \in M$ ).

2. For every ideal $I$ of $L, a(p I)=I$.

3. The pair of complete vector lattices $L$ and $M$ is paired with respect to the natural bilinear functional.

Proof. The hypothesis that $U \subset M$ implies that sup $U=M$ when $U \leqq f$ and $f \in M$ means first that $M$ is a complete vector lattice in its natural order. In addition, if $x \geqq 0$ and $U$ is a subset of $M$ which is directed upwards and has sup $U=f_{0} \in M$, then by the definition of the supremum of a family of linear functionals, $f(x)=\sup f_{\alpha}(x)$ for $f_{\alpha} \in U$. This easily implies that $f(x)$ is an $o$-continuous linear functional of $f$ for each positive $x \in L$ (see, for instance, [4, Corollary 5.7]). But each element of $L$ is the difference of positive elements of $L$, hence also induces an $o$-continuous linear functional on $M$. The hypothesis that $M$ separates points of $L$ implies that the pairing of $M$ and $L$ corresponding to $\langle f, x\rangle=f(x)$ satisfies all of the first definition except perhaps the requirement that the lattice operations already given on $L$ coincide with the lattice operations on $L$ as a set of linear functionals on $M$. Suppose 1 holds. Then if $x>0, x \in c I$, there exists a positive $f \in M$ such that $f(x)>0$. Since the functional $g$, defined by $f$ on $c(I), 0$ on $I$ satisfies $0 \leqq g \leqq f, g \in M$. Then $g \in p I$, $g(x) \neq 0$ whence $x$ is not in $a(p I)$. Thus $a(p I) \subset I$, but the reverse inclusion is trivial so $a(p I)=I$.

Let 2 hold. We need only show that $L$ is a sublattice of the lattice of bounded linear functionals on $M$ to have 3 . Now the hypothesis along with Lemma 3 , implies that $p$ is a $1-1$ order inverting map of the ideals of $L$ onto the ideals of $M$, with inverse $a$. Let $I$ be the ideal $\{y:|y| \wedge(x \vee 0)=0\}$ of $L$. Then $x \wedge 0 \in I$ and $x \vee 0 \in c I$. For positive 
$f$ in $p I, x^{*}(f)=(x \vee 0)^{*}(f)+(x \wedge 0)^{*}(f)=(x \vee 0)^{*}(f) \geqq 0$, while for positive $f \in p(c I), x^{*}(f)=(x \wedge 0)^{*}(f) \leqq 0$. Since $p I \cap p(c I)=(0), p I \cup p(c I)$ $=M$, the positive ideal of $x^{*}$ is contained in $p I$, while its negative ideal is contained in $p(c I)$. Thus $x^{*} \vee 0$ is defined as $x^{*}$ on $p I, 0$ on $p(c I)$ by Lemma 2. Therefore $(x \vee 0)^{*}=x^{*} \vee 0$, and 3 is true.

If 3 is true, Theorem 1 shows that every $o$-continuous linear functional on $L$ bounded by functionals of $M$ is induced by an element of $M$. This is order convexity, so that 1 holds.

Although our results are for complete vector lattices, the following result on completion of a vector lattice enables us to apply some of the results to arbitrary vector lattices.

CoROllary 1. Let $X$ be a vector lattice, let $Y$ be the complete vector lattice of all bounded linear functionals on $X$ and let $Z$ be the complete vector lattice of all o-continuous linear functionals on $Y$. The only $o$-continuous linear functionals on $Z$ are those induced by elements of $Y$, so that there is a 1-1 correspondence between bounded linear functionals on $X$ and o-continuous linear functionals on $Z$.

This follows easily from Theorem 1 and Proposition 1 . We remark that the natural imbedding of $X$ in $Z$ preserves the linear and finite lattice operations.

Applications. First we sketch a proof of the reflexivity of the real function spaces $L_{p}(m), p>1$, where $m$ is an arbitrary countably additive measure. (See [5].) We start from the following version of the monotone convergence theorem: if $f_{1} \leqq f_{2} \leqq \cdots$, is a sequence of summable functions such that $\int f_{n}$ is bounded, then $f=\mathrm{V} f_{n}$, the pointwise supremum, is integrable and $\int f=\lim \int f_{n}$. Now consider a rising set of elements in $L_{p},\left\{x_{\alpha} \mid \alpha \in A\right\}$ such that $S=\sup \left\{\left\|x_{\alpha}\right\|: \alpha \in A\right\}$ exists. By choosing an arbitrary rising subsequence $y_{n}$ with $\lim \left\|y_{n}\right\|$ $=S$, and taking its supremum, we have the supremum $x$ of the set $\left\{x_{\alpha}: \alpha \in A\right\}$. Further $\lim \left\|x_{\alpha}-x\right\|=0$. This implies immediately that both $L_{p}$ and $L_{q}$ for $p^{-1}+q^{-1}=1$ are complete vector lattices in which order convergence implies norm convergence. The Hölder inequality in an obvious calculation shows that $L_{q}$ is a complete lattice of $o$ continuous linear functionals on $L_{p}$, the natural norm and order in $L_{q}$ coinciding with those it has as a lattice of $o$-continuous and norm continuous linear functionals on a Banach lattice $L_{p}$. Any positive norm continuous functional on $L_{\boldsymbol{q}}$ is $o$-continuous, and hence is the pointwise limit of a rising set of linear functionals induced by elements of $L_{p}$. But this set must be bounded in norm by $\|f\|$, and hence has a supremum in $L_{p}$. Thus the positive elements, and hence all the elements of $L_{\boldsymbol{q}}$ are induced by elements of $L_{\boldsymbol{p}}$. 
The duality theory for $L_{1}(m)$ and $L_{\infty}(m)$ is more complicated. Let us give a definition of $L_{\infty}$ suggested by the definition of measurability in $[4$, p. 65]. Let us consider the class of bounded functions which are measurable on every set of finite measure, modulo the subset consisting of those functions the integral of whose absolute value is zero on every such finite set. Choose $\|f\|$ to be the supremum over all sets $S$ of finite measure of the ordinary $L_{\infty}$ norm. This normed linear space we call $L_{\infty}(m)$; of course, this agrees with the ordinary definition for $\sigma$-finite measure spaces. The example quoted in [6, p. 280] along with our theorem below shows that $L_{\infty}$ need not be a complete vector lattice or the dual of $L_{1}$. Let us call the following property of a measure space the Radon-Nikodym property: if $m^{\prime}$ is a measure defined for the same sets as $m$, satisfying $m^{\prime}(B) \leqq C m(B)$ for some constant $C$, there exists an element $f$ of $L_{\infty}$ such that $m^{\prime}(B)=\int_{B} f d m$. It is easy to see that this property is equivalent to the classical Radon Nikodym property. Such a measure $m^{\prime}$ induces a function $M^{\prime}$ on characteristic functions by the equation $m^{\prime}(E)=M^{\prime}\left(\chi_{E}\right)$, if $\chi_{E}$ is the characteristic function of $E$. $M^{\prime}$ has an unique extension $F$ to $L_{1}(m)$, and $F$ satisfies $\|F\| \leqq C$. Now it is also easy to see that the RadonNikodym property is equivalent to the following one, which we call the Riesz property: if $F$ is a bounded linear functional on $L_{1}(m)$, there exists an element $f \in L_{\infty}(m)$ such that $F(g)=\int g f d m$ for $g \in L_{1}$. The following theorem is obviously inspired by Theorem 5.1 of [7].

THEOREM. For any measure the following are equivalent:

1. The Radon-Nikodym property holds.

2. The Riesz property holds.

3. $L_{\infty}$ is a complete vector lattice in its natural order.

4. Every o-continuous linear functional on $L_{\infty}$ is induced by an element of $L_{1}$.

Proof. We have already remarked on the equivalence of 1 and 2 . If 2 is true, then $L_{\infty}$ is complete by the comment on the completeness of a dual space which was made in the introduction. Conversely, if 3 is true, Theorem 1 applies and a positive bounded linear function $F$ on $L_{1}$ is the limit of a rising set of elements of $L_{\infty}$ which must be bounded in norm by $\|F\|$. Hence $F \in L_{\infty}$, and 2 follows clearly. If 3 is true, a positive linear $o$-continuous functional $H$ on $L_{\infty}$ is a supremum of a rising set of elements of $L_{1}$ again by Theorem 1 . The rising set is bounded by $\|H\|$, hence $H \in L_{1}$ and 4 holds; but the hypothesis of 4 implicitly assumes 3 .

The following theorem is mainly due to Vulih [8], who improved some work of Ogasawara and Nakamura in this direction. It should be noticed that this theorem cannot be easily used to imply the 
reflexivity of the $L_{p}$ spaces, since its hypothesis involves a condition on the dual space.

Theorem. Let $L$ be a Banach lattice, $L^{*}$ its dual. In order that $L$ be reflexive, it is necessary and sufficient that both the following be true. $L$ satisfies (a): If $x_{\alpha}$ is a rising set with $\left\|x_{\alpha}\right\| \leqq B$, then sup $x_{\alpha}$ exists. Both $L$ and $L^{*}$ satisfy $(\mathrm{b}): x_{\alpha}$ a decreasing set with infimum 0 implies $\lim \left\|x_{\alpha}\right\|$ $=0$.

Proof. Condition (b) for a complete Banach lattice $L$ is equivalent to the condition that every element of $L^{*}$ be $o$-continuous, by Dini's theorem applied to the function $f \rightarrow f\left(x_{\alpha}\right), f$ in the intersection of the unit sphere and the positive cone of $L$.

Necessity. If $L$ is reflexive, $L$ is the dual of a Banach lattice and hence (a) trivially follows. The evaluation functional is an $o$-continuous functional on $L$, as was remarked in the introduction. Thus every element in $L^{*}$ is $o$-continuous, and (b) follows for $L$. By symmetry (b) follows for $L^{*}$; (a) is trivial.

Sufficiency. We now know that $L^{*}$ is a complete vector lattice of $o$-continuous functionals on $L$ satisfying (b). Any norm continuous linear functional on $L^{*}$ is $o$-continuous then, and is the difference of two positive elements. Condition (a) for $L$ and our theorem now show that $L=L^{* *}$.

REMARK. As a final application, let us mention the theorem that a commutative *-algebra $A$ of operators on a Hilbert space which contains the supremum of every bounded rising net in it, is closed in the strongest operator topology, and hence in the weak topology. A quick proof of this may be had by remarking that the Hermitian elements of $A$ form a complete vector lattice. The strongest topology is the topology of eventually bounded strong convergence, and for a *-algebra, it is well known that closure in this topology is equivalent to closure in the weak topology. In addition the strongest continuous linear functionals are $o$-continuous. A simple argument inspired by one of Dixmier, [3, Theorem 2] completes the proof. This theorem has recently been announced for arbitrary *-algebras of operators, and a generalization due to Segal [6, Theorem 14] of the RadonNikodym theorem gives hope for an extension of our theorem to complete partially ordered spaces which are not lattices. It is true that analogs of Lemmas 1, 2, and 3 hold for the Hermitian elements of a $W^{*}$ algebra, using the appropriate definitions of ideals and $o$-convergence. However, using the definition of McShane [4] for $o$-convergence in a complete partially ordered space, we shall construct a class of examples where the lattice requirements of our theorem are 
not met, and the conclusion of the theorem fails. (These examples have an algebraic structure similar to that of a $W^{*}$-algebra, as has been shown in a note appearing in these Proceedings.) ${ }^{2}$ Thus, a generalization of our theorem to arbitrary complete partially ordered vector spaces appears to be possible, but not simple. Let $B$ be any Banach space, and let $L$ be the linear space of all pairs $(b, t)$ where $b \in B, t$ is real. Let $(b, t) \geqq 0$ mean $\|b\| \leqq t$. Then it may be easily seen that any bounded rising net in $L$ has a supremum, and that the set of all $o$-continuous linear functionals on $L$ is the space $M$ constructed from the dual $B^{*}$ of $B$ in the same way, with the same definition of positivity, where $(f, s)(b, t)=f(b)+s t, f \in B^{*}, b \in B$. Any element $k$ of $B^{* *}$ generates a positive $o$-continuous linear functional on $M$ defined by the pair $(k,\|k\|)$, which is a supremum of elements in $L$ if and only if $k$ is an element of $B$.

\section{REFERENCES}

1. G. Birkhoff, Lattice theory, Amer. Math. Soc. Colloquium Publications vol. 9, 2d ed., 1948.

2. S. Bochner and R. S. Phillips, Additive set functions and vector lattices, Ann. of Math. (2) vol. 42 (1941) pp. 316-324.

3. J. Dixmier, Formes linéaires sur un anneau d'operateurs, Bull. Soc. Math. France vol. 81 (1953) pp. 9-39.

4. E. J. McShane, Order-preserving maps and integration processes, Annals of Mathematics Studies, no. 31, 1953.

5. J. Schwartz, A note on the space $L_{p}^{*}$, Proc. Amer. Math. Soc., vol. 2 (1951) pp. 270-275.

6. I. E. Segal, A non-commutative extension of abstract integration, Ann. of Math. (2) vol. 57 (1953) pp. 401-457.

7. —, Equivalences of measure spaces, Amer. J. Math. vol. 73 (1951) pp. 275313.

8. B. Z. Vulih, On imbedding a normed partially ordered space in its second conjugate space, Uspehi Matematixeskih Nauk (N.S.) vol. 9 (1954) pp. 91-99.

UNIVERSITY OF ViRGINIA AND

University of California, Berkeley

2 D. B. Lowdenslager, On postulates for general guantum mechanics, Proc. Amer. Math. Soc. vol. 8 (1957) pp. 88-91. 